\section{$\underset{\substack{\text { hommes } \\ \text { \& migrations }}}{ }$}

\section{Hommes \& migrations}

Revue française de référence sur les dynamiques

migratoires

$1326 \mid 2019$

Londres et ses migrations

\title{
La Galerie des dons, objet muséal total
}

\section{Elisabeth Jolys-Shimells}

\section{(Q) OpenEdition \\ Journals}

\section{Édition électronique}

URL : https://journals.openedition.org/hommesmigrations/10016

DOI : 10.4000/hommesmigrations. 10016

ISSN : 2262-3353

\section{Éditeur}

Musée national de l'histoire de l'immigration

\section{Édition imprimée}

Date de publication : 1 juillet 2019

Pagination : 143-150

ISBN : 978-2-919040-46-9

ISSN : $1142-852 X$

\section{Référence électronique}

Elisabeth Jolys-Shimells, «La Galerie des dons, objet muséal total », Hommes \& migrations [En ligne], 1326 | 2019, mis en ligne le 01 juillet 2019, consulté le 15 janvier 2022. URL : http:// journals.openedition.org/hommesmigrations/10016; DOI : https://doi.org/10.4000/ hommesmigrations.10016 


\title{
La Galerie des dons, objet muséal total
}

\author{
Élisabeth Jolys-Shimells, \\ conservatrice du patrimoine, responsable des collections ethnographiques au Musée national de l'histoire de \\ l'immigration.
}

Manifestant la volonté de constituer un patrimoine participatif et incarné, la Galerie des dons occupe une place importante dans le projet du Musée national de l'histoire de l'immigration (MNHI). Revendiquée comme une spécificité par ses concepteurs, appréciée des publics, elle juxtapose des récits de vie individuels, illustrés par des objets, déposés ou donnés, rendant hommage aux « invisibles» en leur donnant sans réserve la parole, réparant tantôt les blessures d'une histoire familiale difficile, tantôt celles d'une intégration laborieuse. Objet muséal passionnant aux réelles réussites, la Galerie des dons engage à réinterroger ses aspirations initiales, tout comme les catégories et les processus classiques du patrimoine.

nstallée dans la coursive du deuxième étage du Palais de la Porte dorée, la Galerie des dons est un espace d'exposition de près de $400 \mathrm{~m}^{2}$, partie intégrante du parcours permanent du Musée. La Galerie des dons est née de la volonté d'instaurer une dynamique participative dans le projet initial de la Cité nationale de l'histoire de l'immigration (CNHI), du besoin d'acquérir des objets pour les collections patrimoniales et de l'idée de dédier un espace spécifique à cet effet.

Baptisée ainsi dès son ouverture en 2008, la Galerie des dons n'a pourtant abrité, dans ses premières années, que des dépôts, modalité jugée la plus adéquate pour sa souplesse, mais aussi dans une réflexion éthique par rapport au déposant, qui pourrait vouloir récupérer ses objets, après les avoir vus 《 sacralisés 》 par le Musée. À chaque nouvel ajout, une « fenêtre » était dévoilée dans les vitrines, pour mettre en valeur la dynamique d'enrichissement participatif. Effectivement, l'exposition s'enrichissait, mais pas la collection puisqu'il s'agissait de dépôts, malgré le nom de l'espace.

\section{Un concept muséographique à la fois pragmatique et idéaliste}

Dès le départ, la confusion est présente entre exposition - faire connaître son parcours dans une vitrine - et patrimonialisation - faire un don au bénéfice des collections nationales, dont le premier objet est la conservation, parfois au détriment de l'exposition. Ce constat est pleinement conscient et la réflexion 
théorique autour des collections ethnographiques a été abondante et pertinente, menée au premier chef par Fabrice Grognet, ethnologue et premier responsable de la collection?.

Lorsqu'est amorcé le projet de refonte de l'espace de la Galerie des dons, cette ambiguïté est soulevée, décrite et résolue ainsi par Hélène du Mazaubrun, responsable de ce projet: «Le Musée n'était pas le propriétaire de cette collection, mais une sorte de courroie de transmission et de médiation, sans patrimoine constitué. Pourtant, dans l'espace permanent du Musée, une salle intitulée "Galerie des dons" avait été déployée au cœur du bâtiment. Le nom de la galerie était-il donc mal choisi ? L'appellation "Galerie des dépôts" ou "Galerie participative" aurait-elle été plus appropriée? En 2012, le concept a, au contraire, été réaffirmé. Non seulement le nom de cette salle a été conservé, mais la politique d'acquisition a été modifiée, cherchant à faire évoluer les dépôts en dons, afin de faire entrer en collection nationale l'objet, la parole, mais aussi le geste du don. Car le don n'est pas anodin. En plus du changement de propriétaire qu'il opère, il a une fonction symbolique ${ }^{2}$. »

En 2014, la Galerie des dons a été réouverte dans sa nouvelle présentation avec une adresse aux publics on ne peut plus directe : proposez, vous serez exposés. Ont ainsi été installées, à dessein, des vitrines vides, portant l'inscription "C'est ici que vos dons et récits prendront place. »

Cette louable et souhaitable aspiration s'est heurtée aux complexités inhérentes au fonctionnement d'un musée et n'a pu être mise en œuvre. La présentation n'a connu aucune modification de fond, malgré l'existence de propositions de visiteurs, qui ont été reçues et étudiées avec la rigueur nécessaire, conduisant à en écarter certaines, à retenir les autres, mais sans pouvoir les faire aboutir avant plusieurs années. $\mathrm{Si}$, grâce à la qualité des relations humaines, beaucoup de donateurs potentiels ont compris les données de l'équation et en ont pris leur parti - poursuivre leur démarche ou l'abandonner -, il ne faut pas passer sous silence les frustrations engendrées par ce que l'on peut appeler une promesse non tenue. Ce constat a pour objet d'alimenter la réflexion quant aux choix à mener pour l'avenir de la Galerie des dons, non pour pointer des défaillances, car ce serait nier le déterminisme prépondérant du contexte de création de la CNHI et les

1. Voir notamment Fabrice Grognet, « Un exemple des relations musée / ethnologie : la genèse des collections "ethnographiques" du musée de la Cité nationale de l'histoire de l'immigration », in ethnographiques.org, n²4, juillet 2012.

2. Hélène du Mazaubrun, «La parole et le don », in Hommes \& Migrations, n 1300, 2012, pp. 126-131. 
remarquables réalisations des équipes mobilisées. Leur audace et leur engagement profond ont permis de faire naître et perdurer le Musée national de l'histoire de l'immigration comme une institution de référence, à l'utilité sociale et culturelle sans cesse réaffirmée.

\section{Un espace de médiation culturelle pertinent et efficace}

Inaugurée en 2008, réaménagée en 2014, la Galerie des dons se voit renouvelée en 2019. Cette présentation s'appuie sur la muséographie existante, mais développe un discours construit en grande partie autour de nouvelles acquisitions et des conditions de visionnage améliorées pour les témoignages filmés.

Trois facteurs ont motivé la réalisation de cette nouvelle version. En premier lieu, l'état de conservation des textiles et des photographies, représentant une partie importante du parcours, nécessite leur mise en réserve. D'autre part, il paraissait de même incontournable d'exposer les nouvelles donations, dont certaines ont attendu leur concrétisation depuis 2015, date à laquelle les commissions d'acquisitions du musée ont été suspendues. Enfin, la Galerie des dons est un espace de médiation très performant, qui suscite l'intérêt et l'adhésion des publics individuels, et constitue un outil indispensable pour les médiateurs accompagnant des groupes. Ils utilisent notamment la Galerie des dons comme espace introductif depuis la suppression de la première partie de l'exposition permanente en 2018, qui analysait les motivations de l'exil.

Les usages en médiation ont particulièrement guidé la conception de la version 2019 du parcours de la Galerie des dons, dans la limite des contraintes du projet, notamment la réutilisation du mobilier muséographique existant. En découlent notamment la création d'un appel plus signifiant depuis le prologue ${ }^{3}$, pour que l'on comprenne en un coup d'œil la teneur du discours de la galerie, l'introduction d'un sens de visite, la structuration du parcours en quatre thématiques problématisées (deux concernant les motivations d'exil et deux s'intéressant aux modalités de la patrimonialisation des histoires singulières), la mise en perspective systématique des parcours individuels vers une question transversale (par exemple immigration et marché du travail, les mineurs isolés, les faux-papiers et les frontières...), abordée succinctement et de manière factuelle.

《 Toute parole a une intention ${ }^{4} 》$, rappelle Bernardo Toro dans un texte examinant les rapports entre témoignage et fiction, qui souligne réciproquement que toute lecture d'un parcours de migration présente aussi des attentes: critique ou validation du modèle social français, projection d'un désir de dépaysement... C'est la même dynamique qui génère la Galerie des dons. D'un côté, les donateurs, portant chacun, par le biais du récit de vie, un discours, de réparation, de tolérance, de modèle à suivre pour les «nouveaux immigrés ».

3. Situé au deuxième étage, le prologue est le hall distribuant les différents espaces d'exposition du Musée.

4. Bernardo Toro, «Raconter l'immigration : pourquoi la fiction alors que le témoignage suffirait ? », in Paris Ville monde, Paris, éd. Rue Saint Ambroise, 2018, pp. 5-9. 
De l'autre, les visiteurs, qui s'identifient aux parcours exposés, y trouvent la confirmation de la possibilité de s'intégrer en France, quels que soient son passé et les aléas des premiers temps passés dans la société d'accueil.

Dans les faits, on constate que la Galerie des dons est un espace de médiation très performant, tant pour les visites individuelles qu'accompagnées, pour toutes les classes d'âge. Il est à la fois apprécié des publics, car il raconte des histoires sensibles et accessibles, et des médiateurs, car il permet de développer plusieurs points essentiels du discours: aborder les raisons qui poussent les individus à migrer et leurs conséquences particulières, développer certains points de l'histoire de l'immigration, notamment par le biais des communautés d'origine, mais aussi la genèse et le projet de la CHNI et du MNHI. Le parti pris muséographique de personnalisation du découpage du parcours et des dispositifs de monstration (un parcours/une vitrine) est également un outil de communication à l'adresse des donateurs qui peuvent parfois devenir des mécènes ou, le plus souvent, participer activement à la programmation culturelle : tables rondes, visites personnalisées, interventions pédagogiques... Au-delà du moment du don ou de l'exposition, bon nombre de donateurs (ou de déposants) s'impliquent dans les actions du musée, en suivent l'actualité, expriment leurs points de vue, proposent des activités, s'en font les ambassadeurs. En cela, la Galerie des dons a parfaitement rempli la mission qui consiste à créer un lien concret et vivace entre le musée et une partie de ce qu'il est convenu d'appeler la société civile.

À l'institution revient la charge fondamentale et délicate de clarifier et d'anticiper le champ des possibles, le fait que les objets ne seront pas exposés en permanence, que le discours qui les entoure peut varier, que le don entraîne dépossession... Ce dialogue, attentif mais franc, souple et constant mais contraint par le principe de réalité de la vie d'une institution, constitue une spécificité exigeante mais profondément signifiante, qui inscrit ce musée national dans la philosophie écomuséale, dont la modernité ne se dément pas. Cela explique notamment que le MNHI, comme les autres musées de société évoluant entre passé et présent, histoire et mémoire, soit confronté à des défis patrimoniaux posés, dans son cas particulier, par la Galerie des dons.

\section{Un dispositif d'enrichissement des collections légitime mais circonscrit}

La collection du Musée national de l'histoire de l'immigration est divisée en trois domaines: l'art contemporain, l'histoire et l'ethnologie. Ce dernier est fréquemment assimilé à la Galerie des dons, comme si le dispositif expographique et la politique d'acquisition en ethnologie ne faisaient qu'un. Cela s'explique principalement par le fait que l'enjeu symbolique de la Galerie des dons est très fort chez certains publics et très structurant dans l'histoire du Musée. Ce dispositif installe en effet l'établissement dans une posture de modèle de musée participatif, particulièrement original pour un musée national.

Or, si la Galerie des dons expose en effet uniquement des objets rangés dans la catégorie « ethnologie» du Musée, elle n'expose pas que des objets donnés et inventoriés mais aussi des objets mis en dépôt, ce qui, nous l'avons rappelé, 
était la modalité standard de mise à disposition à l'origine. De même, les objets du domaine ethnologie des collections sont exposés dans le parcours permanent «Repères » et au gré des expositions temporaires. D'autre part, à partir de 2020 conséquemment à la refonte du parcours permanent, il est très probable que l'espace aujourd'hui occupé par le dispositif, dont la forme de coursive a donné l'appellation " galerie », ne soit plus affecté à la présentation d'objets de collection, son climat n'étant pas propice à la conservation matérielle des biens. Aujourd'hui, la réflexion est en cours, d'une part, sur l'affectation des espaces, d'autre part, sur la présence d'un espace valorisant des parcours individuels per se, détachés d'un discours plus large. Ainsi, bien que la politique d'acquisition ne puisse être uniquement dépendante des options de valorisation des collections, il est nécessaire de s'interroger sur les conséquences de cet amoindrissement de la visibilité de la démarche de don, présentée par le musée comme la volonté de co-construire le patrimoine de l'histoire et des cultures de l'immigration en France avec la société civile. Dans les choix à venir, il s'agira de concilier la mise en valeur de la dimension participative et l'évaluation pragmatique des moyens de sa mise œuvre, notamment l'espace, le mobilier et les principes graphiques.

Au-delà du rapport entre les publics et le musée, les modalités de collecte des objets qui composent la Galerie des dons sont l'objet d'une collaboration transversale et ouverte au sein même de l'institution, non circonscrite, comme souvent, au seul service de la conservation. Ainsi, le service Réseaux et partenariats a-t-il joué un rôle particulièrement important dans le lancement et le développement des collections ethnographiques. Dans le même temps, la focalisation de la politique d'acquisition sur les parcours de vie de migrants ou de leurs descendants, si elle a répondu, dans une certaine mesure, à un aspect « participatif » de l'ethnologie patrimoniale, n'a pas permis de rassembler un fonds attestant des usages et des représentations collectifs de la société française en lien avec le fait migratoire, qu'il paraît important de constituer. Pour satisfaire aux attendus d'une « collection nationale de référence», il semble indispensable de se mettre au niveau de ce qui a été accompli pour la collection d'art contemporain en matière de pertinence, de cohérence et de singularité.

De plus, cette modalité de collecte se doit d'être extrêmement qualitative du point de vue de la relation du musée au donateur, en raison de la sensibilité 
du matériau collecté (la mémoire individuelle et bien souvent intime) et de la nécessité éthique de bien faire comprendre au donateur les implications d'un don d'objets assorti d'un témoignage au patrimoine national. Ce dernier implique de se dessaisir de la propriété matérielle et d'une grande part du droit de regard sur les utilisations futures de l'ensemble, en contrepartie de l'assurance d'une conservation optimale des biens le plus longtemps possible, et de l'accession à un statut patrimonial équivalent à celui de la Joconde. À l'inverse, cette collecte induit des possibilités d'exploitation complexes pour le Musée, qui doit garder à l'esprit le caractère totalement subjectif de l'information recueillie, de manière à ne pas le considérer comme représentatif d'un phénomène, et de constamment le manier avec une extrême précaution pour respecter la sensibilité de ceux qu'il concerne, les donateurs et leurs proches. Dans la restitution du récit de vie, partie intégrante du don aux côtés des objets, les histoires personnelles ne sont ni questionnées ni vérifiées par le Musée. La perception singulière et les effets de la mémoire sont considérés comme des qualités intrinsèques des témoignages. Mais, lorsqu'il s'agit d'articuler ses histoires dans l'Histoire, le Musée ne peut mettre en présence des éléments contradictoires, sauf à développer une médiation particulière. La mise en dialogue peut s'avérer particulièrement complexe, considérant les effets conjugués de la rigueur scientifique, du respect des personnes et de la réception des publics.

Il ne s'agit pas pour autant d'abandonner la collecte de parcours singuliers, mais celle-ci est déjà aujourd'hui un domaine investi par les musées et autres institutions patrimoniales dites "de société» sur le territoire, ce qui n'était pas le cas au moment de la création de la CNHI (on peut à cette occasion reconnaître les fruits portés par le travail de maillage du territoire effectué ces dix dernières années). D'autre part, la littérature, le cinéma et d'autres formes d'art ont également participé, sous des formes propres, à la collecte et à la conservation de ce type de témoignages. Il n'y a donc pas d'impératif d'exhaustivité ou de péril qui nécessiterait que la MNHI lance des opérations de grande ampleur de collecte d'objets témoignant de parcours individuels. Néanmoins, les enjeux sont multiples : cibler davantage les appels aux dons pour combler les lacunes (géographiques ou historiques notamment), identifier les relais qui permettraient de collecter des parcours de personnages à qui la notoriété confère une envergure nationale ou internationale ainsi que d'identifier les «zones blanches» à défricher: parcours en marge (par exemple personnes transgenres, personnes incarcérées, précaires...), personnalités politiques, associatifs, culture populaire..., dont les parcours ne seront collectés a priori par aucune autre institution muséale ou patrimoniale publique. Si la voix des migrants reste un axe fondamental pour constituer ce secteur de collection, il semble également important de réunir davantage de parcours de personnes non migrantes mais aussi des accueillants, au sens très large (associatifs, agents de l'administration, chercheurs...). En parallèle, il s'agira d'élargir la collection à des ensembles de nature différente, témoignant de faits sociaux en rapport avec l'histoire de l'immigration, anciens ou contemporains. À cet égard, le travail de relecture des collections déjà effectué par bon nombre de musées de 
société territoriaux constitue un référentiel précieux pour orienter les axes d'enrichissement pour le MNHI, qui doit trouver les objets susceptibles de rendre compte de la dimension nationale et spécialisée de son périmètre patrimonial.

\section{Du travail de collecte ethnographique au détour par la fiction}

Il semblerait ainsi que l'appellation de ce secteur de collection doive être remise en débat. Dès l'origine, la collection de ces parcours a été qualifiée d'ethnologique, conformément à l'approche scientifique qui a guidé les principes de patrimonialisation du Musée national de l'histoire de l'immigration, mais aussi la cohérence des profils de ceux qui en ont eu la responsabilité. Son premier responsable, Fabrice Grognet, en dresse un constat très tranché: "Il n'existe pas, en effet, d'objet ayant une nature ontologique spécifique qui le détermine à devenir ethnographique. C'est avant tout le regard d'un scientifique - l'ethnologue qui par son étude en France ou à l'étranger fixe ce qualificatif sur une chose matérielle qui peut préexister à la collecte ou, au contraire, être créée spécialement à cette occasion. Dès lors, l'expression "objet ethnographique" semble correspondre fondamentalement à une catégorie "par défaut", traduisant la position de la démarche ethnologique vis-à-vis des beaux-arts et de l'histoire au sein de la CNHI. Toutefois, la volonté initiale de générer des "mémoires orales", non prises en compte à cet instant par le Musée, semble être une opportunité pour insérer un "regard ethnographique" au cœur du processus et ainsi enrichir - voire dépasser - la mission originelle de pourvoyeur d'objets ${ }^{5}$. 》 Ce point de vue, développé dans les tout premiers temps de la collection, pourrait sans doute être nuancé aujourd'hui, l'inclusion de la mémoire orale, fixée par le texte et la vidéo, faisant partie intégrante du don et de sa contractualisation. D'autre part, si les discussions actuelles penchent vers une qualification de la collection en lien avec les sociétés et l'humain, il ne faudra pas faire l'économie d'une redéfinition globale des collections, notamment pour définir l'articulation avec la collection dite « historique ».

Avec le recul, il semble que l'intention de conduire une démarche ethnologique rigoureuse ait été rendue impossible par la prééminence de l'objet Galerie des dons, qu'elle devait pourtant alimenter. Cela avait d'ailleurs été anticipé par Fabrice Grognet: « $\bar{A}$ bien des égards, et comme le signale Daniel Fabre à propos d'autres collectes à ambition patrimoniale, la collection dite "ethnographique" du musée de la CNHI semble finalement obéir "à une logique qui lui est propre et qui, à mon sens, n'entretient pas de relation nécessaire avec l'ethnologie comme science sociale (...). Ce ne sont pas des faits sociaux qui sont ainsi rassemblés mais des objets précieux que collecteurs et spectateurs reconnaissent immédiatement, avec émotion et plaisir" ${ }^{\prime \prime} . »$

5. Fabrice Grognet, op. cit. Voir également Fabrice Grognet, «Objets de musée, n'avez-vous donc qu'une vie?», in Gradhiva, n² 2, 2005, pp. 49-63.

6. Voir Daniel Fabre, «L'ethnologue et ses sources », in Terrain, $n^{\circ} 7,1986, p p .3-13$, cité par Fabrice Grognet, op. cit. 
Ce qui pourrait apparaître comme une carence pourrait en réalité faire la force de cette collection, à condition d'en assumer le caractère autofictionnel, sans qu'il faille pour autant renoncer à l'idée de sélection et de rigueur. En s'affranchissant de leur contexte initial, il semble possible de s'appuyer à nouveau sur les propos de Bernardo Toro: « seul le passage à la fiction peut libérer le témoignage de sa mise sous tutelle ${ }^{7} »$, qu'il juge d'autant plus grande dans le cas des migrants face à leur société d'accueil. Ce déséquilibre du rapport de force est accru dans le dialogue entre un individu, le postulant donateur, le plus souvent animé par des motivations très personnelles et donc particulièrement sensibles à un jugement de valeur, et l'institution muséale nationale, arbitrant les valeurs «monumentales ${ }^{8}$ » du don proposé, mais qui, bien qu'il s'en défende sincèrement, juge le potentiel exemplaire du parcours pour «la connaissance, le plaisir et l'éducation du public ${ }^{9} »$.

Reprenant la pertinente réflexion éthique menée dès le départ de la constitution de la Galerie des dons, comment concilier respect des individus, exigences d'un musée national et nécessaire construction du corpus matériel à la mise en œuvre, aux côtés de corpus académiques et idéologiques ${ }^{10}$ riches et articulés, d'un dialogue équilibré dans les projets muséaux liés à l'histoire de l'immigration ? L'enjeu crucial que revêt ce dernier point nous conduit à ne pas renoncer aux objets, à leur patrimonialisation. Sans un ensemble stable, documenté, protégé et légitimé par le statut des collections nationales, il semble impossible de sortir de l'usage de l'objet réduit à l'illustration d'un discours, façonné dans un compromis variable entre le scientifique et le politique, au sens large. Sans amoindrir la pertinence des deux autres corpus de références, il semble pourtant que les objets soient porteurs de sens et d'information, intrinsèquement, qu'ils soient indispensables à l'analyse et à la médiatisation d'un sujet aussi aisément manipulable que l'immigration. Dans cette vaste entreprise, aux côtés d'autres objets, les petites légendes de la Galerie des dons peuvent jouer un rôle utile pour rappeler la part sensible et singulière de celles et de ceux qui, s'ils sont des sujets pour le chercheur, des esprits à conquérir pour le militant, sont avant tout les auteurs d'une existence dont le musée n'est que le réceptacle, instrument sacré mais instrument tout de même, d'une version, d'un instantané.

\footnotetext{
7. Bernardo Toro, op. cit., p. 7.

8. Pour reprendre l'analyse faite par Georges Brunel de la théorie d'Aloïs Riegl des Denkmalswerte, «les valeurs qui se rapportent à l'objet en tant que monument, celles qu'il faut prendre en compte quand se pose la question de savoir comment le conserver. Si on ne perd pas de vue le sens de cette expression, se rend compte que le système exposé dans le Denkmalkultus n'est pas un assortiment de concepts destinés au classement des objets, mais un outil fabriqué en vue de l'action que l'on exerce sur eux. Prendre en compte les valeurs monumentales consiste à élucider ses propres idées et invite chacun à assumer ses propres responsabilités. » Georges Brunel, «La foire aux valeurs », CeROArt, hors-série, juin 2015, http ://journals.openedition.org/ ceroart/4639
}

9. Loi du 4 janvier 2002 relative aux musées de France, article premier.

10. Au sens de systèmes d'idées et d'ensemble des représentations issues de l'opinion (individus, collectifs, politique, médias). 